\title{
Botulinum Toxin Treatment Practice Recommendations for Movement Disorders Clinics During the COVID-19 Pandemic
}

\author{
Roland Dominic G. Jamora, MD, 1,2* Jeryl Ritzi T. Yu, MD, ${ }^{3 *}$ Adrian I. Espiritu, MD, 2,4 Ryan M. Llorin, MD, \\ Rosemarie M. Tiongson, $\mathrm{RN}^{6}$ and Arlene R. Ng, MD, $\mathrm{PhD}^{1}$

\begin{abstract}
${ }^{1}$ Movement Disorders Service and Section of Neurology, Institute for Neurosciences, St. Luke's Medical Center, Global City, Philippines
${ }^{2}$ Division of Adult Neurology, Department of Neurosciences, College of Medicine and Philippine General Hospital,

University of the Philippines Manila, Manila, Philippines

${ }^{3}$ Section of Neurology, Institute for Neurosciences, St. Luke's Medical Center, Quezon City, Philippines

${ }^{4}$ Department of Clinical Epidemiology, College of Medicine, University of the Philippines Manila, Manila, Philippines

${ }^{5}$ Infection Control Services and Department of Internal Medicine, St. Luke's Medical Center, Global City, Philippines

${ }^{7}$ Nursing Service and Movement Disorders Service, St. Luke's Medical Center, Global City, Philippines
\end{abstract}

\begin{abstract}
Background. Due to the Coronavirus disease 2019 (COVID-19) pandemic, non-emergency services like botulinum toxin A (BoNT) injections were temporarily discontinued.
\end{abstract}

Objective. We aimed to create standard practices that optimize the management of patients needing BoNT injections while ensuring both patients' and healthcare professionals' safety.

Methods. We evaluated published guidelines and best practices to determine their applicability for BoNT injection during this pandemic. We then devised a decision tree algorithm for use in our hospital.

Results. A decision tree algorithm has been formulated based on patient evaluation and risk stratification, triaging, the urgency of the procedure, and proper precautions on personal protective wear as appropriate for the procedure's risk stratification.

Conclusion. The algorithm is in place to ensure the safety of both the patient and healthcare professional. It can be adopted and revised by other centers to suit their clinic needs.

Key Words: movement disorders, COVID-19, botulinum toxin, safety recommendations, treatment, pandemic

\section{INTRODUCTION}

The total number of confirmed COVID-19 cases from Severe Acute Respiratory Syndrome coronavirus 2 (SARS-CoV-2) in the Philippines is 507,717, with 10,116 deaths. ${ }^{1}$ Patients typically experience fever, colds, respiratory symptoms, and diarrhea and may also manifest with neurologic symptoms. ${ }^{2,3}$ Due to the first surge of cases in March 2020, an enhanced community quarantine (ECQ)

${ }^{*}$ Dr. Jamora and Dr. Yu shared first authorship for this paper.

Corresponding author: Roland Dominic G. Jamora, MD Department of Neurosciences

College of Medicine and Philippine General Hospital

University of the Philppines Manila

Taft Avenue, Ermita, Manila 1000, Philippines

Email: rgjamora@up.edu.ph was declared. During this time, resources were shifted mainly to care for COVID-19 related medical conditions. Healthcare facilities quickly adapted by temporarily discontinuing face-to-face clinic consultations and limiting non-urgent services, including non-emergency surgical procedures. ${ }^{4-6}$ However, services not related to COVID-19, such as botulinum toxin $\mathrm{A}$ injections (BoNT) for neurologic 
conditions, are still essential. ${ }^{7}$ BoNT treatment sessions have been postponed during the ECQ, and most of these patients received treatment sessions beyond the efficacy window for BoNT injections. ${ }^{8-10}$

As the government started easing the quarantine, outpatient clinics gradually resumed with guidelines in place. ${ }^{11,12}$ However, in-hospital clinics that provide services requiring face-to-face consult, such as the movement disorders clinic, continue to operate with a lean workforce. Given our local setting's current situation, we formulated safety strategies and treatment algorithms in the movement clinic for patients needing BoNT injections during this COVID-19 pandemic based on local and international guidelines.

\section{METHODS}

We reviewed the relevant literature available from the US National Library of Medicine databases and websites using PubMed, World Health Organization, US Centers for Disease Control and Prevention (CDC), the Philippine Department of Health, and the Philippine Society for Microbiology and Infectious Diseases. This was created based on the evaluations of published guidelines and best practices during the COVID-19 pandemic and their applicability in the performance of BoNT injections in the movement disorders clinic. A comprehensive search was conducted employing the key search terms "coronavirus disease 2019", "COVID-19", "SARS-CoV-2," and "botulinum toxin" until July 18, 2020. We further hand-searched the reference list of relevant articles. Three authors (JRTY, AIE, RMT) reviewed the full text of relevant articles identified. The following manuscript was circulated to other experts (RDGJ, RML, ARN), who critically appraised the evidence and revised the report's final version. A proposed clinical management pathway was generated derived from the literature review and experience of the authors.

\section{Patient and Public Involvement}

Patients were not involved in the design and conduct of this research. However, the clinical management pathway generated will be applied to the patients undergoing BoNT injections.

\section{CLINICAL PRACTICES AND ALGORITHM}

\section{Patient evaluation}

\section{Screening and Triaging}

Patients needing BoNT injections are advised to coordinate with the movement disorders clinic nurse to schedule a preliminary screening via online consult to reduce exposure (Figure 1). ${ }^{11,12}$ Consent is secured before the session. The physician sees the patient via telemedicine. ${ }^{13}$ During the remote assessment session, all pertinent details of the clinical history are gathered to allot treatment sessions solely for BoNT injections and minimize exposure during the actual clinic visit.

The patients and accompanying caregivers are screened for any symptoms. Those with fever, colds, respiratory symptoms, and malaise will be redirected to the appropriate facility., ${ }^{2,14}$ The procedure will then be scheduled at a later time. Those who have been exposed to a COVID-19 positive case within the past 14 days or with a history of travel to a COVID-19 known positive cluster within the past 14 days are advised to present a COVID-19 negative swab result before scheduling. ${ }^{15}$

Patients who are free of COVID-19 symptoms are scheduled based on the urgency of the procedure. Urgency is based on the following (a) loss of range of motion due to severe pain related to patient's symptoms, (b) deteriorating daily function due to new or severe symptoms, and (c) the last injection beyond the past three months based on the duration of efficacy. ${ }^{5}$

If none of these criteria are fulfilled, the patient is advised to schedule the procedure later. These patients can be re-assessed as needed. Patients who necessitate urgent treatment are stratified based on their underlying medical conditions that increase their risk of developing severe illness from COVID-19.16 Since comorbid conditions have shown evidence to result in poor recovery from COVID-19 infection, these must be taken into consideration when scheduling patients for in-hospital administration of BoNT injections.

\section{High-risk patients}

The following are classified as high-risk based on the level of evidence as stipulated by CDC recommendations: serious cardiac conditions including heart failure, coronary artery disease or cardiomyopathies, chronic obstructive pulmonary disease, chronic kidney disease, obesity (BMI >30), sickle cell disease, solid organ transplantation, type 2 diabetes mellitus. ${ }^{16}$ For patients stratified as high-risk but necessitating urgent treatment, medical management as an outpatient should be maximized. However, those without relief of symptoms and still opt to undergo the procedure are asked to sign a waiver.

\section{Low- to moderate-risk patients}

Patients with the following are stratified as low- to moderate-risk: asthma, cerebrovascular disease, hypertension, pregnancy, smoking, use of corticosteroids or other immunosuppressive medications, bone marrow transplantation, human immunodeficiency virus infection, immune deficiencies, inherited metabolic disorders, neurologic conditions, other chronic lung diseases, liver disease, type 1 diabetes mellitus, thalassemia. ${ }^{17}$ These patients are scheduled for a procedure. Safety protocols and procedures for visits to the health facility will be detailed to the patient. 


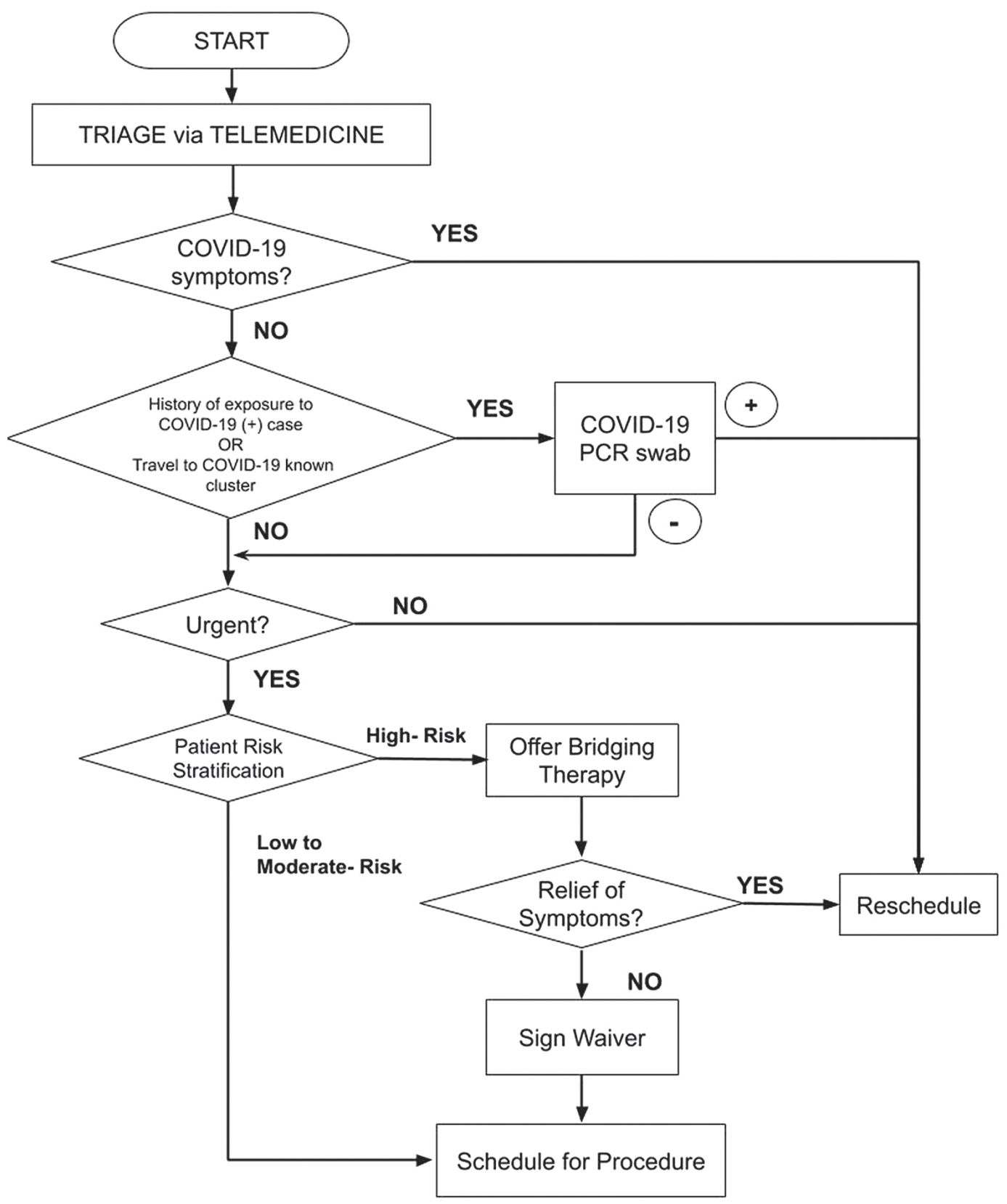

Figure 1. The algorithm on eligibility for in-hospital movement disorders clinic botulinum toxin injections.

\section{Procedure scheduling}

The movement disorders clinic in our institution is located within the Neurosciences Complex, where the other units (such as neurophysiology, memory, brain tumor, etc.) are situated. Since these units share a common waiting area, the number of patients to be scheduled is coordinated with other units/services to ensure social distancing in the shared waiting area and to limit the number of patients in the center at any given time. ${ }^{12}$ The average turnaround time for patients receiving BoNT injections in our clinic is 30 minutes. No more than four patients are scheduled per half day to ensure adequate social distancing and time for preparation, sanitation, and decontamination before and after procedures. For more complex dystonias, an online consultation will provide a venue for preliminary screening. During the face-to-face consult, a more detailed and focused assessment can be done before the BoNT injection. This minimizes exposure during the face-to-face consult by shortening conversation time while allowing the physician to focus on physical examination.

Patients scheduled for procedures are oriented by the clinic staff in line with institutional safety practices. The health declaration form and appointment slip are emailed to the patient before the procedure. They are instructed 
to fill up the said form and bring both documents on the scheduled procedure date.

Instructions for patients before arriving at the health facility

- Patients are instructed to arrive 15 minutes before their scheduled appointment to allow screening while maintaining patient flow inside the health facility.

- The health facility has designated points of entry. Before entry, a temperature check is done. The appointment slip and health declaration form are presented.

- Once in the Central Triage screening area, they are screened for COVID-19 symptoms. If with any symptoms, the procedure is deferred, and they are advised to consult at the emergency room.

- Screening personnel wear masks, eye protection, and fluid-repellent gown and maintain a distance of at least one meter from patients. ${ }^{17}$

- The following are strictly implemented within hospital premises $^{11,17}$ via information panels and hospital staff reminders: "no face mask and no face shield, no entry" policy, practice physical distancing, observe hand hygiene, and proper cough and sneeze etiquette.

- After completing the screening process, the patient proceeds to the movement clinic 5 minutes before the scheduled appointment to limit exposure in the clinic waiting room.

\section{Risk classification of the procedure and personal protective equipment (PPE)}

Clinical assessment pre-procedure allows us to practice minimizing conversations with the patient during procedures, contributing to a reduction in exposure time. However, the proximity of the healthcare professional to the patient during BoNT injections still potentially increases the probability of transmission.

The general practice assumes that all patients may be asymptomatic COVID-19 carriers until such a time when routine SARS-CoV-2 reverse transcription-polymerase chain reaction (RT-PCR) test is part of the initial screening process. ${ }^{18}$ Procedures are classified as low, moderate, or high-risk based on the risk of transmitting the virus to healthcare professionals. These include areas injected which factor in the feasibility of the patient to be masked during the procedure, duration of the procedure, and the potential for aerosolization..$^{6,19}$

\section{High-risk procedures}

Procedures with a risk of aerosolization and those exposed to oral mucosa and secretions are classified as highrisk procedures (Figure 2). In our clinic, any procedure that involves injection via intraoral or percutaneous approach with the jaw open with or without the guidance of electromyography is classified as high-risk. Adequate PPE includes sterile, disposable gloves, surgical cap, eye protection, N95 mask, face shield, shoe cover, and a fluid repellent gown. ${ }^{6,19}$

\section{Moderate-risk procedures}

Injections performed on the middle to lower a third of the face prohibits using a patient's mask and are classified as moderate-risk. ${ }^{19}$ These include injections for hemifacial spasm and ultrasound-guided injection of the parotids. As a safety practice, patients are advised not to talk during the procedure without the mask on. Adequate PPE is mandatory. ${ }^{6}, 19$

\section{Low-risk procedures}

Injections on the upper third of the face and extrafacial sites such as cervical muscles, limbs, etc., are classified as low-risk. These include injections for blepharospasm, selected dystonia cases such as cervical, focal limb dystonia, truncal dystonia, etc., and limb spasticity. Adequate PPE is mandatory. ${ }^{6,19}$

\section{Safety practices and staffing in our movement} disorders clinic

- Patients are instructed to arrive 15 minutes before the scheduled procedure to limit the number of patients in the waiting room. Chairs in the waiting area are arranged to allow a distance of 2 meters between each patient. ${ }^{17}$ Only patients requiring special assistance (elderly, minorities, persons with disability) may bring one companion who is instructed to stay in the waiting area while the procedure is ongoing.

- Separate rooms are dedicated to BoNT injections, paperwork, and the personal belongings of staff. Diagnostic equipment and room furniture are ergonomically positioned in the procedure room.

- During the treatment session, only the physician and a nurse should stay inside the procedure room. The room contains only essential items to limit transmission of the virus via fomites on non-essential items.

- To reduce the spread of pathogens, hospital ventilation measures through clinic vents are regularly monitored by the engineering department to maintain a wellventilated clinic. ${ }^{11,17}$ Additional measures such as HighEfficiency Particulate Air filters are installed to reduce infectious aerosols within the confined area. ${ }^{11,17,20}$

- BoNT is reconstituted before patient entry into the procedure room to minimize exposure time.

- The staff is constantly trained on COVID-19 preventive strategies and safety protocols. The health status of the team is routinely monitored.

- Patients are instructed to adhere to designated entry and exit points to maintain one-way flow for patients within the Neurosciences Complex..$^{11}$ Patients are oriented on hygiene rules via information panels and reminders by the clinic staff to minimize viral spread risk. 


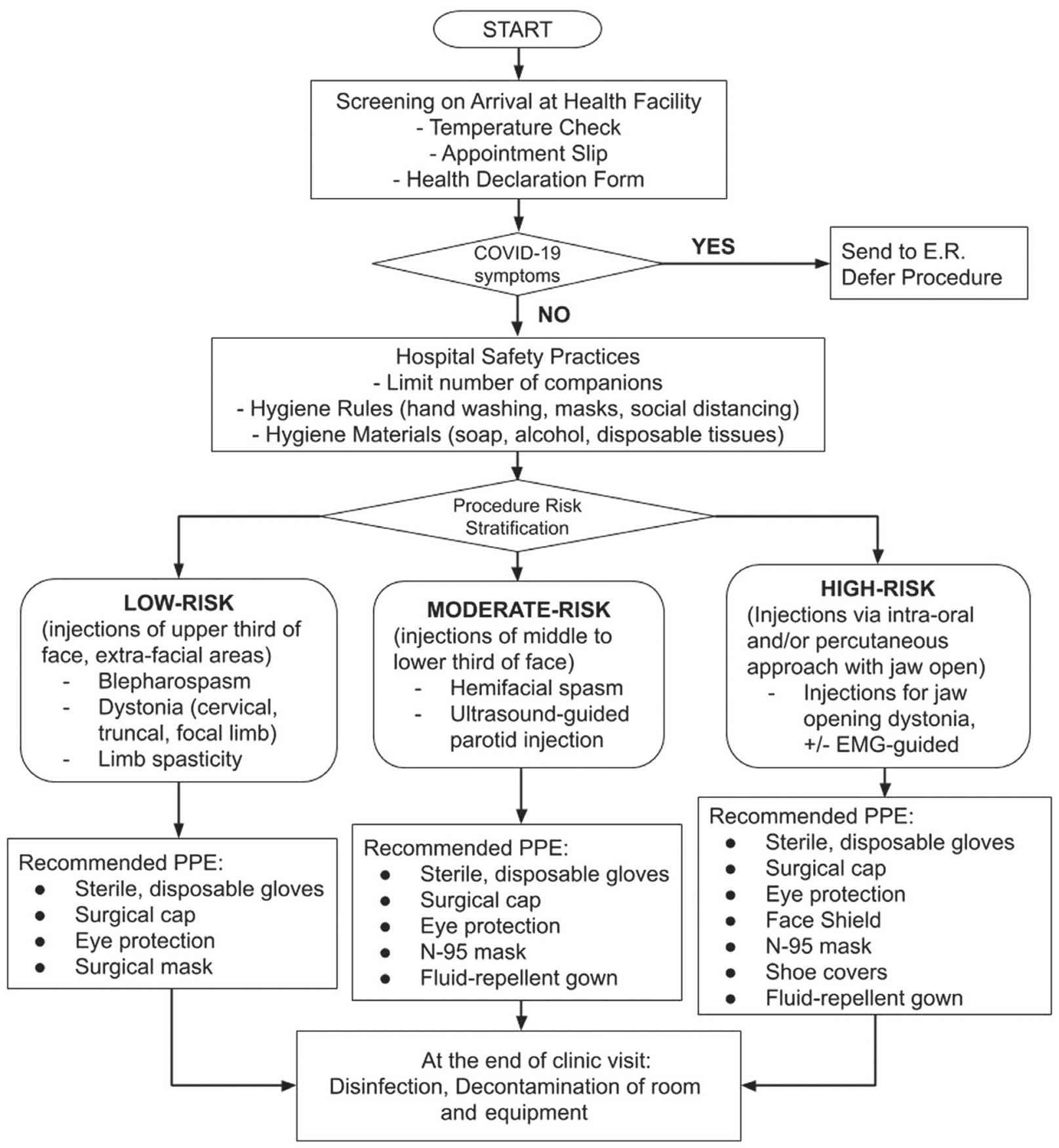

Figure 2. Algorithm describing safety practices and precautions for hospital and movement disorders clinic team members.

- Since COVID-19 may also be transmitted via contact and fomites, it has been shown to survive for as long as 72 hours on specific surfaces; rational sterilization principles are practiced. ${ }^{21}$ Frequently touched surfaces in the room and noncritical items such as the linen, bed, BP apparatus, and stethoscope are decontaminated, cleaned, and disinfected before and after each treatment session. ${ }^{22}$ Disinfection is done using a $70 \%$ or higher concentration of alcohol-based sanitizer or sodium hypochlorite three to four times a day. ${ }^{23}$

\section{CONCLUSION}

During this pandemic, it is essential to optimize management for patients requiring BoNT injections while ensuring patient and healthcare professionals' safety. We emphasize proper triaging of patients to identify those needing face-to-face treatment in the movement clinic. Remote assessment via telemedicine is incorporated in our clinical practices to triage and assess patients while providing management for non-urgent cases. This algorithm may be 
adjusted to accommodate more patients as the staff gets used to the flow and better control of the COVID-19 infection.

\section{Statement of Authorship}

All authors participated in data collection and analysis, and approved the final version submitted.

\section{Author Disclosure}

All authors declared no conflicts of interest.

\section{Funding Source}

No funding support.

\section{REFERENCES}

1. Department of Health, Covid-19 tracker - Philippines. 2021 [cited $2021 \mathrm{Jan}]$. Available from: https://doh.gov.ph/covid19tracker.

2. Guan W, Ni Z, Hu Y, Liang WH, Ou CQ, He JX, et al. Clinical characteristics of coronavirus disease 2019 in China. N Engl J Med. 2020;382(18):1708-1720. https://doi.org/10.1056/NEJMoa2002032

3. Collantes MEV, Espiritu AI, Sy MCC, Anlacan VMM, Jamora RDG. Neurological manifestations in COVID-19 infection: a systematic review and meta-analysis. Can J Neurol Sci. 2021;48(1): 66-76. https://doi.org/10.1017/cjn.2020.146

4. Boldrini P, Bernetti A, Fiore P. Impact of COVID-19 outbreak on rehabilitation services and Physical and Rehabilitation Medicine physicians' activities in Italy. An official document of the Italian PRM Society (SIMFER). Eur J Phys Rehabil Med. 2020;56(3):316-8. https://doi.org/10.23736/S1973-9087.20.06256-5.

5. Baricich A, Santamato A, Picelli A, Morone G, Smania N, Paolucci S, et al. Spasticity treatment during COVID-19 pandemic: clinical recommendations. Front Neurol. 2020;11:719. https://doi.org/ 10.3389/fneur.2020.00719

6. Philippine Society of Microbiology and Infectious Diseases, Risk assessment of surgeries in the context of COVID-19. 2020 [cited 2021 Jan]. Available from: https://www.psmid.org/risk-assessmentof-surgeries-in-the-context-of-covid-19/.

7. Simpson DM, Blitzer A, Brashear A, Comella C, Dubinsky $\mathrm{R}$, Hallet $\mathrm{M}$, et al. Assessment: botulinum neurotoxin for the treatment of movement disorders (an evidence-based review). Neurology. 2008;70(19):1699-1706. https://doi.org/10.1212/01.wnl. 0000311389.26145 .95

8. Nigam P, Nigam A. Botulinum toxin. Indian J Dermatol. 2010;55: 8-14. https://doi.org/10.4103/0019-5154.60343

9. Dressler D, Saberi FA, Barbosa ER. Botulinum toxin: mechanisms of action. Arq Neuropsiquiatr. 2005;63:180-185. https://doi.org/10.1590/ S0004-282X2005000100035

10. Pajo AT, Espiritu AI, Jamora RD. Impact and challenges of the COVID-19 pandemic on patients requiring botulinum toxin treatment. J Mov Disord. 2021;14(1):29-33. https://doi.org/10.14802/jmd.20088
11. Philippine Society of Microbiology and Infectious Diseases, Infection prevention and control guidelines for outpatient clinic resumption in the context of COVID-19. 2020 [cited 2021 Jan]. Available from: https://www.psmid.org/infection-prevention-and-control-guidelinesfor-outpatient-clinic-resumption-in-the-context-of-covid-19/.

12. Centers for Disease Control and Prevention, Healthcare facilities: managing operations during the COVID-19 pandemic. 2020 [cited $2021 \mathrm{Jan}]$. Avialable from: https://www.cdc.gov/coronavirus/2019ncov/hcp/guidance-hcf.html.

13. Centers for Disease Control and Prevention, Using telehealth to expand access to essential health services during the COVID-19 pandemic. 2020 [cited $2021 \mathrm{Jan}$ ]. Available from: https://www.cdc. gov/coronavirus/2019-ncov/hcp/telehealth.html.

14. Li Q, Guan X, Wu P, Wang X, Zhou L, Tong Y, et al. Early transmission dynamics in Wuhan, China of novel coronavirus-infected pneumonia. N Engl J Med. 2020;382(13):1199-1207. https://doi.org/10.1056/ NEJMoa2001316

15. Fineberg HV. Ten weeks to crush the curve. N Engl J Med. 2020;382:e37. https://doi.org/10.1056/NEJMe2007263

16. Centers for Disease Control and Prevention, Evidence used to update the list of underlying medical conditions that increase a person's risk of severe illness from COVID-19. 2020 [cited 2021 Jan]. Available from: https://www.cdc.gov/coronavirus/2019-ncov/need-extraprecautions/evidence-table.html.

17. World Health Organization, Infection prevention and control during health care when coronavirus disease (COVID-19) is suspected or confirmed- interim guidance. 2020 [cited 2021 Jan]. Available from: https://www.who.int/publications/i/item/WHO-2019-nCoVIPC-2020.4.

18. Bai Y, Yao L, Wei T, Tian F, Jin DY, Chen L, et al. Presumed asymptomatic carrier transmission of COVID-19. JAMA 2020;323(14):1406-1407. https://doi.org/10.1001/jama.2020.2565.

19. Kapoor KM, Chatrath V, Boxley SG, Nurlin I, Snozzi P, Demosthenous $\mathrm{N}$, et al. COVID-19 pandemic: consensus guidelines for preferred practices in an aesthetic clinic. Dermatol Ther. 2020;33(4):e13597. https://doi.ogr/10.1111/dth.13597

20. Wen Z, Yang W, Li N, Wang J, Hu J, Li J, et al. Assessment of the risk of infectious aerosols leaking to the environment from BSL3 laboratory HEPA air filtration systems using model bacterial aerosols. Particuology. 2014;13:82-87. https://doi.org/10.1016/j. partic.2012.11.009.

21. van Doremalen N, Bushmaker T, Morris DH, Holbrook MG, Gamble A, Williamson BN, et al. Aerosol and surface stability of SARSCoV-2 as compared with SARS-CoV-1. N Engl J Med. 2020;382(16) 1564-1567. https://doi.org/10.1056/NEJMc2004973

22. Centers for Disease Control and Prevention, A rational approach to disinfection and sterilization. 2008 [cited 2021 Jan]. Available from: https://www.cdc.gov/infectioncontrol/guidelines/disinfection/ rational-approach.html.

23. Kampf G, Todt D, Pfaender S, Steinmann E. Persistence of coronaviruses on inanimate surfaces and their inactivation with biocidal agents. J Hosp Infect. 2020;104:246-251. https://doi.org/ 10.1016/j.jhin.2020.01.022 